\title{
Two-temperature dual-phase-lags theory in a thermoelastic solid half-space due to an inclined load
}

\author{
Ashraf M. Zenkour ${ }^{1,2}$, Ahmed E. Abouelregal ${ }^{3,4}$, Khaled A. Alnefaie ${ }^{5}$, Nidal H. Abu-Hamdeh ${ }^{5}$, \\ Abdulmalik A. Aljinaidi ${ }^{5}$, and Elias C. Aifantis ${ }^{5,6,7,8}$ \\ ${ }^{1}$ Department of Mathematics, Faculty of Science, King Abdulaziz University, P.O. Box 80203, \\ Jeddah 21589, Saudi Arabia \\ ${ }^{2}$ Department of Mathematics, Faculty of Science, Kafrelsheikh University, Kafrelsheikh 33516, Egypt \\ ${ }^{3}$ Department of Mathematics, College of Science and Arts, University of Aljouf, El-Qurayat, Saudi Arabia \\ ${ }^{4}$ Department of Mathematics, Faculty of Science, Mansoura University, Mansoura 35516, Egypt \\ ${ }^{5}$ Department of Mechanical Engineering, Faculty of Engineering, King Abdulaziz University, \\ Jeddah 21589, Saudi Arabia \\ ${ }^{6}$ School of Mechanics and Engineering, Southwest Jiaotong University, Chengdu, China \\ ${ }^{7}$ Laboratory of Mechanics and Materials, Polytechnic School, Aristotle University of Thessaloniki, \\ Thessaloniki, Greece \\ ${ }^{8}$ Michigan Technological University, Houghton, Michigan, USA and ITMO University, St. Petersburg, Russia \\ Correspondence to: A. M. Zenkour (zenkour@gmail.com)
}

Received: 21 October 2015 - Revised: 27 June 2016 - Accepted: 20 July 2016 - Published: 22 August 2016

Abstract. This article addresses the thermoelastic interaction due to inclined load on a homogeneous isotropic half-space in context of two-temperature generalized theory of thermoelasticity with dual-phase-lags. It is assumed that the inclined load is a linear combination of both normal and tangential loads. The governing equations are solved by using the normal mode analysis. The variations of the displacement, stress, conductive temperature, and thermodynamic temperature distributions with the horizontal distance have been shown graphically. Results of some earlier workers have also been deduced from the present investigation as special cases. Some comparisons are graphically presented to estimate the effects of the two-temperature parameter, the dual-phaselags parameters and the inclination angle. It is noticed that there is a significant difference in the values of the studied fields for different value of the angle of inclination. The method presented here maybe applicable to a wide range of problems in thermodynamics and thermoelasticity.

\section{Introduction}

As it is well known the equation of heat conduction of classical uncoupled theory of thermoelasticity does not contain any elastic terms and this leads to that the elastic changes have no effect on temperature. In addition, the heat equation is of a parabolic type, predicting infinite speed of propagation for heat waves. The above two phenomena that predicted by the classical uncoupled theory of thermoelasticity are not compatible with physical observations. So, the theory of coupled thermoelasticity has been formulated by Biot (1956) to eliminate the first paradox inherent in the classical uncoupled theory. However, the heat equations for both coupled and un- coupled theories of the diffusion type predict infinite speeds of propagation for heat waves contrary to physical observations. Additional two generalized thermoelasticity theories are presented by Lord and Shulman (1967) and Green and Lindsay (1972) to eliminate the second paradox inherent in the classical uncoupled and coupled theories. They two generalized thermoelasticity theories treat the heat propagation as a wave phenomenon rather than a diffusion phenomenon and predict a finite speed of heat propagation.

Green and Naghdi (1993) have presented another generalized theory of thermoelasticity without energy dissipation. This theory differs from the previous theories in that it does 
not accommodate dissipation of thermal energy and it includes the isothermal displacement gradients among its independent constitutive variables. Zenkour (2015) has presented the exact 3D solutions for the field quantities of thermal shock plate problem. A unified generalized thermoelasticity theory is presented for the transient thermal shock plate problem in the context of the generalized and coupled thermoelasticity theories.

Tzou (1995a, 2014) has proposed a new model which is known as the dual-phase-lag (DPL) model. The physical meanings and the applicability of the DPL mode have been supported by the experimental results of Tzou (1995b). Chandrasekharaiah (1986) and Tzou (1995b) have proposed another DPL model to modify the classical thermoelastic one. They presented an approximation to a modified Fourier law with two different time translations: a PL of the heat flux $\tau_{\mathrm{q}}$ and a PL of the temperature gradient $\tau_{\theta}$ (see also, Ignaczak and Ostoja-Starzewski, 2010). Zenkour et al. (2013) have presented the effect of DPL model on reflection of thermoelastic waves in a solid half-space with variable material properties. Zenkour and Abouelregal (2015) have presented the effects of PLs in a thermoviscoelastic orthotropic continuum with a cylindrical hole and variable thermal conductivity.

The heat conduction of the two-temperature theory of thermoelasticity (2TT) in a deformable media depends upon two distinct temperatures. The first is the conducting temperature and the second is the thermodynamic temperature. The 2TT is considered as one of the non-classical theories of elastic solids with thermal dependence. For time-independent problems, the difference between these two temperatures is proportional to the heat supply. If the heat supply is ignored, the two temperatures are identical. However, for time-dependent situations and for wave propagation problems in particular, the two temperatures are in general different, regardless of the presence of a heat supply. Chen and Gurtin (1968) and Chen et al. (1969) have presented such a theory involving a non-simple material for which the two temperatures are not identical. The two temperatures and the strain are found to have representation in the form of a travelling wave plus a response, which occur instantaneously throughout the body (see Warren and Chen, 1973). Recently, Zenkour and Abouelregal (2014) have presented the state-space approach for an infinite medium with a spherical cavity based upon generalized 2TT. Abbas and Zenkour (2014a) have used the finite element method to investigate a DPL model on thermoelastic interactions in a semi-infinite medium under a ramp-type heating. Zenkour (2016) has presented a 2$\mathrm{D}$ coupled solution for thermoelastic beams via generalized DPLs model. Abbas and Zenkour (2014b) have presented 2TT for generalized thermoelastic interaction in an infinite fiber-reinforced anisotropic plate containing a circular cavity with two relaxation times. Abouelregal and Zenkour (2016) have presented the generalized thermoelastic interactions due to an inclined load at a two-temperature half-space.
The deformation in thermoelastic half-space due to inclined loads is the subject of many investigators. Kumar and Rani (2005) have studied the deformation due to inclined load in thermoelastic half-space with voids. Othman et al. (2009) have studied 2-D problems in a half-space possesses cubic symmetry as a result of inclined load. Kumar and Gupta (2010) have investigated the deformation in an orthotropic micropolar thermoelastic solid with two relaxation times as a result of the inclined load. Sharma (2011) has used the integral transform technique to study the deformation in inhomogeneous, isotropic thermodiffusion elastic half-space as a result of inclined load. Ailawalia and Kumar (2010) have obtained the analytic formulations for the field quantities on the free surface of micropolar thermoelastic medium possessing cubic symmetry with one relaxation time as a result of time harmonic inclined load.

Our main aim in writing this paper is to present thermoelastic interaction due to inclined load on a clamped boundary of a half-space in the context of two-temperature generalized thermoelasticity with DPLs. The analytic expressions for the displacement, stress, and temperature distributions on the free surface of thermoelastic solid as a result of inclined load have been obtained. Some comparisons have been shown in figures to estimate the effect of the inclination angle and the two-temperature parameter on all the studied fields.

\section{Basic equations}

The field equations for a linear, homogeneous and isotropic thermoelastic material, in the context of generalized thermoelasticity with DPLs and two temperatures and in the absence of the body force, take the following forms:

Equations of motion:

$\mu \nabla^{2} \boldsymbol{u}+(\lambda+\mu) \nabla(\operatorname{div} \mathbf{u})-\gamma \nabla \theta=\rho \ddot{u}$.

The constitutive equations:

$\boldsymbol{\sigma}=\lambda(\operatorname{div} \mathbf{u}) \mathbf{I}+\mu\left[\nabla \mathbf{u}+(\nabla \mathbf{u})^{\mathrm{Tr}}\right]-\gamma \theta \mathbf{I}$.

The strain-displacement relations:

$e_{i j}=\frac{1}{2}\left(u_{i, j}+u_{j, i}\right)$.

The heat conduction equation:

$$
\begin{aligned}
& \left(1+\tau_{\theta} \frac{\partial}{\partial t}\right) \nabla^{2}\left(K \phi_{, i}\right)_{, i} \\
& =\left(\delta+\tau_{q} \frac{\partial}{\partial t}\right)\left(\rho C_{E} \frac{\partial \theta}{\partial t}+\gamma T_{0} \frac{\partial e_{k k}}{\partial t}-Q\right) .
\end{aligned}
$$

The above equation is the same as the DPL model of Tzou and 2TT. It is to be noted that the direct vector or tensor notation is employed in the above equations. Also, an over dot denotes the partial derivative with respect to the time $t$. In 
Eqs. (1)-(4), $\boldsymbol{u}$ is the displacement vector, $\theta=T-T_{0}$ denote the thermodynamical temperature, $T_{0}$ is the reference temperature, $\gamma=(3 \lambda+2 \mu) \alpha_{t}, \alpha_{t}$ is the coefficient of volume expansion, $\lambda$ and $\mu$ are the Lame' constants, $\rho$ is the mass density, $\sigma$ is the stress tensor, $\mathbf{I}$ is the identity tensor and the suffix $\mathrm{Tr}$ is the transpose of the given vector. In addition, $e_{i j}$ is the strain tensor, $e_{k k}=e$ is the cubical dilatation, $K$ is the thermal conductivity, $C_{E}$ is the specific heat at constant strain, $Q$ is the heat supplied per unit volume from the external work, $\tau_{\theta}$ is the PL of the heat flux, $\tau_{q}$ is PL of temperature gradient and $0 \leq \tau_{\theta}<\tau_{q}$, which will ensure that the heat conduction equation will predict finite speeds of heat propagation, and $\phi$ is the conductive temperature measured from the temperature $\phi_{0}$, and satisfies the relation

$\theta=\phi-a \phi_{, i i}$,

where $a$ is the two-temperature parameter (temperature discrepancy) and $\phi_{0}=T_{0}$, is the reference temperature. The coupled thermoelasticity theory, generalized thermoelasticity theory with one relaxation time, and the generalized theory without energy dissipation follow as limited cases depending on the value of $\delta, \tau_{q}$, and $\tau_{\theta}$.

Putting $\tau_{\theta}=0, \delta=1$, and $\tau_{q}=\tau_{0}$ (the first relaxation time), gives the fundamental equations possible for Lord and Shulman's (LS) theory. Putting $\tau_{\theta}=\delta=0$ gives Green and Naghdi's (GN) model. In the absence of phase-lag of the heat flux and phase-lag of gradient of temperature, Eq. (2) reduces to equation for a classical thermoelastic body (CTE).

\section{Statement of the problem}

A homogeneous thermoelastic half-space with two temperatures occupying the region $x \geq 0$ is considered. The $z$ axis is taken perpendicular to the bounding plane pointing inwards. It is assumed that the initial state of the medium is quiescent. The surface of the medium is subjected to an inclined load. This load is assumed to be a linear combination of normal and tangential loads. The present study is restricted to the plane strain parallel to $x z$ plane. The problem is thus twodimensional in which the field quantities are functions of the spatial variables $x$ and $z$ as well as on the time variable $t$.

The displacement vector $\boldsymbol{u}$, thus, has the form $\boldsymbol{u}=$ $(u, 0, w)$. For the 2-D problem in the $x z$-plane, Eq. (1) can be re-written as

$(\lambda+2 \mu) \frac{\partial^{2} u}{\partial x^{2}}+(\lambda+\mu) \frac{\partial^{2} w}{\partial x \partial z}+\mu \frac{\partial^{2} u}{\partial z^{2}}-\gamma \frac{\partial \theta}{\partial x}=\rho \frac{\partial^{2} u}{\partial t^{2}}$,
$(\lambda+2 \mu) \frac{\partial^{2} w}{\partial z^{2}}+(\lambda+\mu) \frac{\partial^{2} u}{\partial x \partial z}+\mu \frac{\partial^{2} w}{\partial x^{2}}-\gamma \frac{\partial \theta}{\partial z}=\rho \frac{\partial^{2} w}{\partial t^{2}}$,

The generalized equation of heat conduction, Eq. (4), without heat source, is re-written in the $x z$-plane as

$K\left(1+\tau_{\theta} \frac{\partial}{\partial t}\right)\left(\frac{\partial^{2} \phi}{\partial x^{2}}+\frac{\partial^{2} \phi}{\partial z^{2}}\right)$

$$
=\left(\delta+\tau_{q} \frac{\partial}{\partial t}\right)\left(\rho C_{E} \frac{\partial \theta}{\partial t}+\gamma T_{0} \frac{\partial e}{\partial t}\right),
$$

and Eq. (5) becomes

$\theta-\phi=-a\left(\frac{\partial^{2} \phi}{\partial x^{2}}+\frac{\partial^{2} \phi}{\partial z^{2}}\right)$.

Beside to the above equations we can deal with the following constitutive relations:

$\sigma_{x x}=(\lambda+2 \mu) \frac{\partial u}{\partial x}+\lambda \frac{\partial w}{\partial z}-\gamma \theta$,

$\sigma_{y y}=\lambda\left(\frac{\partial u}{\partial x}+\frac{\partial w}{\partial z}\right)-\gamma \theta$,

$\sigma_{z z}=(\lambda+2 \mu) \frac{\partial w}{\partial z}+\lambda \frac{\partial u}{\partial x}-\gamma \theta$,

$\sigma_{z x}=\mu\left(\frac{\partial u}{\partial z}+\frac{\partial w}{\partial x}\right)$.

\section{Solution of the problem}

Let us define the two displacement potentials $\Phi$ and $\Psi$ which are related to the displacements $u$ and $w$ as,

$u=\frac{\partial \Phi}{\partial x}-\frac{\partial \Psi}{\partial z}, \quad w=\frac{\partial \Phi}{\partial x}+\frac{\partial \Psi}{\partial z}$.

So, the governing equations become

$$
\begin{aligned}
& c_{1}^{2} \nabla^{2} \Phi-\frac{\partial^{2} \Phi}{\partial t^{2}}=\frac{\gamma}{\rho}\left(\phi-a \nabla^{2} \phi\right), \\
& c_{2}^{2} \nabla^{2} \Psi-\frac{\partial^{2} \Psi}{\partial t^{2}}=0, \\
& K\left(1+\tau_{\theta} \frac{\partial}{\partial t}\right) \nabla^{2} \phi=\left(\delta+\tau_{q} \frac{\partial}{\partial t}\right)\left[\rho C_{E} \frac{\partial}{\partial t}\left(\phi-a \nabla^{2} \phi\right)\right. \\
& \left.+\gamma T_{0} \frac{\partial}{\partial t}\left(\nabla^{2} \Phi\right)\right] \\
& \sigma_{x x}=\lambda \nabla^{2} \Phi+2 \mu \frac{\partial}{\partial x}\left(\frac{\partial \Phi}{\partial x}-\frac{\partial \Psi}{\partial z}\right)-\gamma\left(\phi-a \nabla^{2} \phi\right), \\
& \sigma_{y y}=\lambda \nabla^{2} \Phi-\gamma\left(\phi-a \nabla^{2} \phi\right) \text {, } \\
& \sigma_{z z}=\lambda \nabla^{2} \Phi+2 \mu \frac{\partial}{\partial z}\left(\frac{\partial \Psi}{\partial x}+\frac{\partial \Phi}{\partial z}\right)-\gamma\left(\phi-a \nabla^{2} \phi\right), \\
& \sigma_{z x}=2 \mu \frac{\partial^{2} \Phi}{\partial x \partial z}+\mu\left(\frac{\partial^{2} \Psi}{\partial x^{2}}-\frac{\partial^{2} \Psi}{\partial z^{2}}\right),
\end{aligned}
$$

where

$$
\begin{aligned}
c_{1}^{2} & =\frac{\lambda+2 \mu}{\rho}, \\
c_{2}^{2} & =\frac{\mu}{\rho}, \\
\nabla^{2} & =\frac{\partial^{2}}{\partial x^{2}}+\frac{\partial^{2}}{\partial z^{2}} .
\end{aligned}
$$


For the purpose of numerical evaluation, the following dimensionless variables are introduced:

$$
\begin{array}{r}
\left\{x^{\prime}, z^{\prime}, u^{\prime}, w^{\prime}\right\}=\frac{\eta_{0}}{c_{1}}\{x, z, u, w\},\left\{t^{\prime}, \tau_{0}^{\prime}, \tau_{\theta}^{\prime}, \tau_{q}^{\prime}\right\} \\
=\eta_{0}\left\{t, \tau_{0}, \tau_{\theta}, \tau_{q}\right\}, \quad \eta_{0}=\frac{\rho C_{E} c_{1}^{2}}{K} \\
\left\{\Phi^{\prime}, \Psi^{\prime}, a^{\prime}\right\}=\left(\frac{\eta_{0}}{c_{1}}\right)^{2}\{\Phi, \Psi, a\}, \\
\left\{\theta^{\prime}, \phi^{\prime}\right\}=\frac{\gamma}{\rho c_{1}^{2}}\{\theta, \phi\}, \quad \sigma_{i j}^{\prime}=\frac{\sigma_{i j}}{\mu} .
\end{array}
$$

Using the dimensionless variables given above in Eqs. (15)(17) (after removing the primes), one obtains

$$
\begin{aligned}
& \nabla^{2} \Phi-\frac{\partial^{2} \Phi}{\partial t^{2}}=\phi-a \nabla^{2} \phi \\
& \nabla^{2} \Psi-\beta^{2} \frac{\partial^{2} \Psi}{\partial t^{2}}=0 \\
& {\left[\left(1+\tau_{\theta} \frac{\partial}{\partial t}\right)+\eta_{0} a \frac{\partial}{\partial t}\left(1+\tau_{0} \frac{\partial}{\partial t}\right)\right] \nabla^{2} \phi} \\
& =\eta_{0}\left(\delta+\tau_{q} \frac{\partial}{\partial t}\right)\left[\frac{\partial \phi}{\partial t}+\varepsilon \frac{\partial}{\partial t}\left(\nabla^{2} \Phi\right)\right],
\end{aligned}
$$

where

$\varepsilon=\frac{\gamma^{2} T_{0}}{\rho C_{E}(\lambda+2 \mu)}, \quad \beta^{2}=\frac{\lambda+2 \mu}{\mu}$,

Note that $\varepsilon$ represents the dimensionless thermoelastic coupling constant while $\beta^{2}$ is the ratio of the longitudinal waves speed to the shear waves speed.

The transverse normal stress as well as the tangential shear stress given in Eqs. (20) and (21) become

$$
\begin{aligned}
\sigma_{z z} & =\left(1-2 \beta^{2}\right) \nabla^{2} \Phi+2 \frac{\partial}{\partial z}\left(\frac{\partial \Psi}{\partial x}+\frac{\partial \Phi}{\partial z}\right) \\
& -\beta^{2}\left(\phi-a \nabla^{2} \phi\right), \\
\sigma_{z x} & =2 \frac{\partial^{2} \Phi}{\partial x \partial z}+\frac{\partial^{2} \Psi}{\partial x^{2}}-\frac{\partial^{2} \Psi}{\partial z^{2}} .
\end{aligned}
$$

\section{Normal mode analysis}

Cheng and Zhang (2000) have proposed the normal mode expansion method for modelling the thermoelastic generation process of elastic waveforms in an isotropic plate. Allam et al. (2009) have used this method to study the 2-D problem of electromagneto-thermoelasticity for a homogeneous isotropic perfectly conducting thick plate subjected to a timedependent heat source in the context of Green and Naghdi's thermoelasticity theory.

The solution of the field variables can be decomposed in terms of normal modes and are given in the following form (Allam et al., 2009):

$\left\{u, w, \phi, \Phi, \Psi, \sigma_{i j}\right\}(x, z, t)$

$$
=\left\{u^{*}, w^{*}, \phi^{*}, \Phi^{*}, \Psi^{*}, \sigma_{i j}^{*}\right\}(x) \mathrm{e}^{\omega t+\mathrm{i} \zeta z}
$$

where $\omega$ is the (complex) frequency, $\mathrm{i}=\sqrt{-1}, \zeta$ is the wave number in the $z$-direction, and $u^{*}(x), w^{*}(x), \phi^{*}(x), \Phi^{*}(x)$, $\Psi^{*}(x)$, and $\sigma_{i j}^{*}(x)$ are the amplitudes of the field variables. Using Eq. (30), then Eqs. (24)-(26) take the form

$$
\begin{aligned}
& \left(D^{2}-\alpha_{1}\right) \Phi^{*}=-a\left(D^{2}-\alpha_{2}\right) \phi^{*} \\
& q \varepsilon\left(D^{2}-\zeta^{2}\right) \Phi^{*}=\left(D^{2}-\alpha_{3}\right) \phi^{*} \\
& \left(D^{2}-k_{3}^{2}\right) \Psi^{*}=0
\end{aligned}
$$

where

$$
\begin{aligned}
& \alpha_{1}=\zeta^{2}+\omega^{2}, \quad \alpha_{2}=\zeta^{2}+\frac{1}{a}, \\
& \alpha_{3}=\zeta^{2}+q, \quad k_{3}^{2}=\zeta^{2}+\omega^{2} \beta^{2}, \\
& q=\frac{\omega \eta_{0}\left(1+\tau_{0} \omega\right)}{1+\tau_{\theta} \omega+\omega \eta_{0} a\left(1+\tau_{0} \omega\right)}, \quad D=\frac{\mathrm{d}}{\mathrm{d} x} .
\end{aligned}
$$

Eliminating $\phi^{*}(x)$ or $\Phi^{*}(x)$ in Eqs. (31) and (32), one gets the following fourth-order differential equation for $\phi^{*}(x)$ or $\Phi^{*}(x)$ :

$$
\left(D^{4}-A D^{2}+B\right)\left\{\phi^{*}(x), \Phi^{*}(x)\right\}=0,
$$

where

$$
A=\frac{\alpha_{1}+\alpha_{3}-q \varepsilon a\left(\zeta^{2}+\alpha_{2}\right)}{1-q \varepsilon a}, \quad B=\frac{\alpha_{1} \alpha_{3}-q \varepsilon a \zeta^{2} \alpha_{2}}{1-q \varepsilon a} .
$$

Introducing $k_{i}(i=1,2,3)$ into Eq. (35), one can obtain

$$
\left(D^{2}-k_{1}^{2}\right)\left(D^{2}-k_{2}^{2}\right)\left\{\phi^{*}(x), \Phi^{*}(x)\right\}=0,
$$

where $k_{1}^{2}$ and $k_{2}^{2}$ are the roots of the characteristic equation

$k^{4}-A k^{2}+B=0$.

The roots of the above equation are given by

$k_{1}^{2}=\frac{1}{2}\left[2 A+\sqrt{A^{2}-4 B}\right]$,

$k_{2}^{2}=\frac{1}{2}\left[2 A-\sqrt{A^{2}-4 B}\right]$.

Keeping in mind that $\phi^{*}, \Phi^{*} \rightarrow 0$ as $x \rightarrow \infty$ for surface waves, the solution of Eq. (37) has the form

$\left\{\phi^{*}(x), \Phi^{*}(x)\right\}=\sum_{n=1}^{2}\left\{m_{n}(a, \omega), M_{n}(a, \omega)\right\} \mathrm{e}^{-k_{n} x}$,

where $m_{n}(a, \omega)$ and $M_{n}(a, \omega)$ are specific functions depending on $a$ and $\omega$. Substituting Eq. (40) into Eq. (32), one obtains the following relation:

$M_{n}(a, \omega)=H_{n} m_{n}(a, \omega), \quad n=1,2$, 
where

$H_{n}=\frac{k_{n}^{2}-\alpha_{3}}{q \varepsilon\left(k_{n}^{2}-\zeta^{2}\right)}, \quad n=1,2$.

Thus, one has

$\left\{\phi^{*}(x), \Phi^{*}(x)\right\}=\sum_{n=1}^{2}\left\{1, H_{n}\right\} m_{n}(a, \omega) \mathrm{e}^{-k_{n} x}$.

From the regularity condition, one obtains

$\Psi^{*}(x)=m_{3}(a, \omega) \mathrm{e}^{-k_{3} x}$

where $m_{3}(a, \omega)$ is specific function depending on $a$ and $\omega$. Making use of solutions given in Eqs. (43) and (44), one gets

$$
\begin{aligned}
& u^{*}=-\sum_{n=1}^{2} k_{n} H_{n} m_{n}(a, \omega) \mathrm{e}^{-k_{n} x}-\mathrm{i} \zeta m_{3}(a, \omega) \mathrm{e}^{-k_{3} x} \\
& w^{*}=\mathrm{i} \zeta \sum_{n=1}^{2} H_{n} m_{n}(a, \omega) \mathrm{e}^{-k_{n} x}-k_{3} m_{3}(a, \omega) \mathrm{e}^{-k_{3} x}
\end{aligned}
$$

Using Eqs. (9) and (30), one obtains

$\theta^{*}=\sum_{n=1}^{2}\left[\left(1+a \zeta^{2}\right)-a k_{n}^{2}\right] m_{n}(a, \omega) \mathrm{e}^{-k_{n} x}$.

Substituting Eqs. (43) and (44) into Eqs. (28) and (29) with the aid of Eq. (30), one gets

$$
\begin{aligned}
& \sigma_{z z}^{*}=\sum_{n=1}^{2} L_{n} m_{n}(a, \omega) \mathrm{e}^{-k_{n} x}-2 \mathrm{i} \zeta m_{3}(a, \omega) \mathrm{e}^{-k_{3} x}, \\
& \sigma_{z x}^{*}=-2 \mathrm{i} \zeta \sum_{n=1}^{2} k_{n} H_{n} m_{n}(a, \omega) \mathrm{e}^{-k_{n} x}+\left(\zeta^{2}+k_{3}^{2}\right) m_{3}(a, \omega) \mathrm{e}^{-k_{3} x},
\end{aligned}
$$

where

$$
\begin{aligned}
L_{n} & =\left[-2 \zeta^{2}+\left(1-2 \beta^{2}\right)\left(k_{n}^{2}-\zeta^{2}\right)\right] H_{n} \\
& -\beta^{2}\left[1-a\left(k_{n}^{2}-\zeta^{2}\right)\right] .
\end{aligned}
$$

\section{Applications}

Here, the parameters $m_{j}(a, \omega)(j=1,2,3)$ will be determined exactly. In the physical problem, we should suppress the positive exponentials that are unbounded at infinity. Suppose that the inclined line load $F_{0}$ is acting along the interface on the $x$ axis and its inclination with $z$ axis is $\theta_{0}$.

Let us consider the normal line load $F_{1}$ acting in the positive $x$ direction on the surface $x=0$ along the $z$ axis and the tangential load $F_{2}$ is acting at the origin in the positive $x$ direction. So, the boundary conditions on the surface $y=0$ are

$\left\{\sigma_{z z}, \sigma_{z x}\right\}(0, z, t)=-\left\{F_{1}, F_{2}\right\}(x) \mathrm{e}^{\omega t+\mathrm{i} \zeta z}$, where for the inclined line load $F_{0}$ we have $F_{1}=F_{0} \cos \theta_{0}$ and $F_{2}=F_{0} \sin \theta_{0}$.

The surface $x=0$ is thermally insulated, i.e., it satisfies the boundary condition

$\frac{\partial \phi(0, z, t)}{\partial x}=0$.

The substitution of the field quantities into the above boundary conditions, yields

$$
\begin{aligned}
& \sum_{n=1}^{2} L_{n} m_{n}(a, \omega)-2 \mathrm{i} \zeta k_{3} m_{3}(a, \omega)=-F_{1}, \\
& -2 \mathrm{i} \zeta \sum_{n=1}^{2} k_{n} L_{n} m_{n}(a, \omega)+\left(\zeta^{2}+k_{3}^{2}\right) m_{3}(a, \omega)=-F_{2}, \\
& \sum_{n=1}^{2} k_{n} m_{n}(a, \omega)=0
\end{aligned}
$$

or in the matrix form

$$
\left\{\begin{array}{c}
m_{1} \\
m_{2} \\
m_{3}
\end{array}\right\}(a, \omega)=\left[\begin{array}{ccc}
L_{1} & L_{2} & -2 \mathrm{i} \zeta k_{3} \\
-2 \mathrm{i} \zeta k_{1} H_{1} & -2 \mathrm{i} \zeta k_{2} H_{2} & \zeta^{2}+k_{3}^{2} \\
k_{1} & k_{2} & 0
\end{array}\right]^{-1}\left\{\begin{array}{c}
-F_{1} \\
-F_{2} \\
0
\end{array}\right\} .
$$

After determining the parameters $m_{j}(a, \omega)$, we can deduce the expressions for all field quantities of the medium.

Finally, a number of special cases of different thermoelasticity theory are relevant to document here:

- The generalized one-temperature thermoelasticitytheory (1TT) maybe obtained when $a \rightarrow 0$.

- The coupled thermoelasticity (CTE) theory maybe obtained when $\tau_{q}=\tau_{\theta}=0$ and $\delta=1$.

- The Lord-Shulman (LS) generalized thermoelasticity theory maybe obtained when $\tau_{\theta}>0, \delta=1$ and $\tau_{q}=$ $t_{0}>0$.

- The generalized thermoelasticity without energy dissipation (the linearized GN theory of type II) is obtained when $\tau_{\theta} \rightarrow 0, \delta=0$ and $\tau_{q}=1$.

\section{Numerical results}

For the sake of completeness we present some numerical applications to illustrate the analytical procedure presented earlier. The results depict the variations of the two temperatures (thermodynamic $\theta$ and conductive $\phi$ ), the in-plane displacement $u$, the transverse deflection $w$, the transverse normal stress $\sigma_{z z}$, and the tangential shear stress $\sigma_{z x}$.

Copper material is chosen to evaluat the numerical results. The Cooper material properties of the present problem in SI units (Allam et al., 2009) are given by

$$
\begin{aligned}
& K=368 \mathrm{Wm}^{-1} \mathrm{~K}^{-1}, \quad \alpha_{t}=1.78 \times 10^{-5} \mathrm{~K}^{-1}, \\
& C_{E}=383.1 \mathrm{~J} \mathrm{~kg}^{-1} \mathrm{~K}^{-1}, a=2,
\end{aligned}
$$




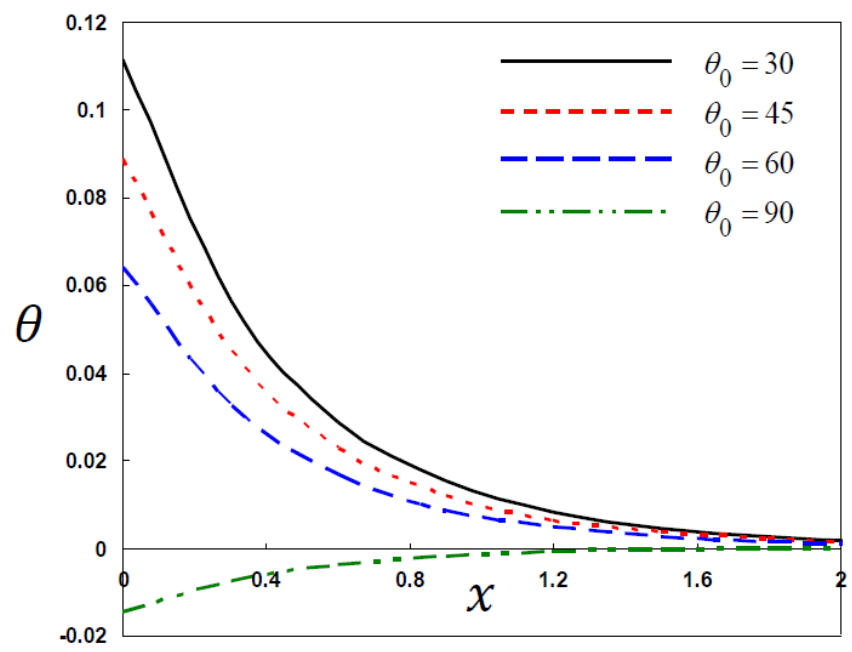

Figure 1. Variation of thermodynamic temperature $\theta$ with distance $x$ for different values of the inclination angle $\theta_{0}$.

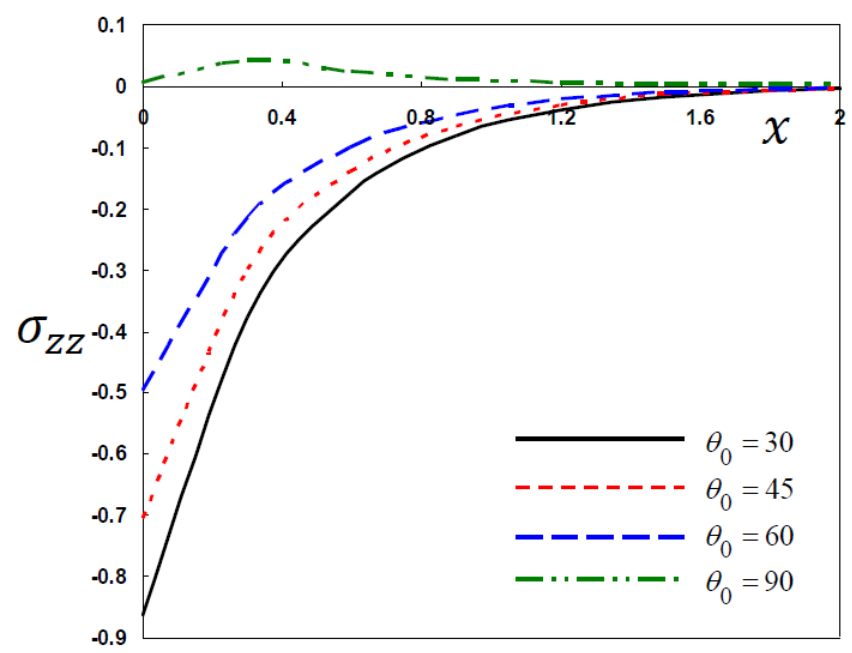

Figure 2. Variation of transverse normal stress $\sigma_{z z}$ with distance $x$ for different values of the inclination angle $\theta_{0}$.

$\rho=8954 \mathrm{~kg} \mathrm{~m}^{-3}, \quad \lambda=7.76 \times 10^{10} \mathrm{kgm}^{-1} \mathrm{~s}^{-2}$,

$\mu=3.86 \times 10^{10} \mathrm{kgm}^{-1} \mathrm{~s}^{-2}$,

$\beta^{2}=4, \quad T_{0}=293 \mathrm{~K}, \quad \varepsilon=0.0168$.

The computations are carried out on the surface $z=1$ at $t=0.15$. The graphically results for the distribution of the real part of the dimensionless field quantities are shown in Figs. 1-7 with $F_{0}=1, \omega=\omega_{0}+\mathrm{i} \xi, \omega_{0}=2, \xi=0.1$ and $\zeta=2.1$ (Kumar and Rani, 2005). All the field quantities are evaluated inside the medium on the $z$ axis as functions of $x$.

Figures 1 and 2 give comparisons of the thermodynamic temperature $\theta$ and the transverse normal stress $\sigma_{z z}$ against the direction $x$ for different values of angle of inclination $\theta_{0}$ (orientation of source) and the two-temperature parameter remains constant $a=0.2$. It is seen that the angle of inclination

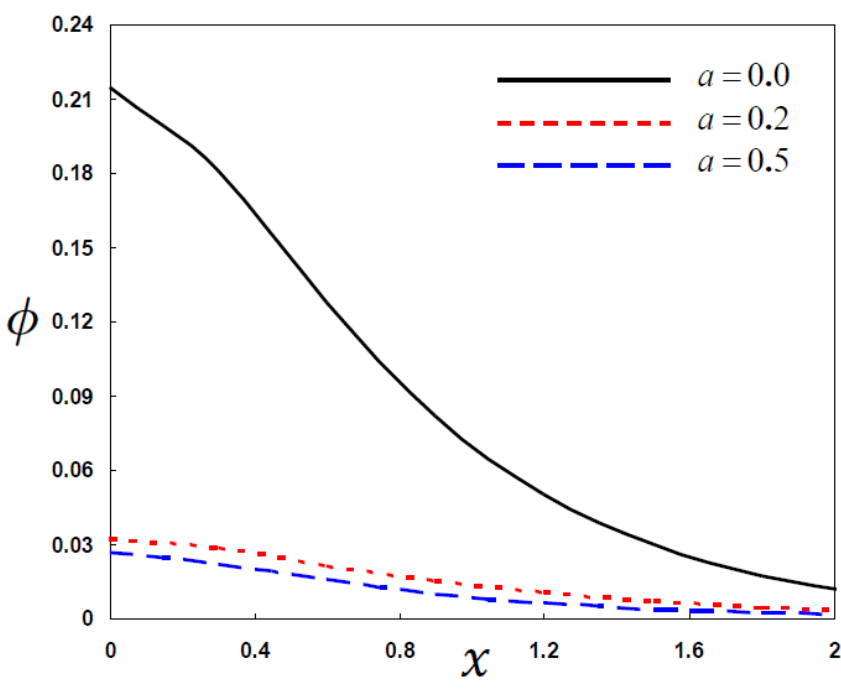

Figure 3. Variation of conductive temperature $\phi$ with distance $x$ for different values of the two-temperature parameter $a$.

$\theta_{0}$ has significant effects on the two studied fields. It plays a vital role on the development of temperature and stress fields in which the following points are observed:

- Significant difference in the values of the studied fields is noticed for different value of the inclination angle $\theta_{0}=0,30,45,60$ and 90 .

- As $\theta_{0}$ increases the values of the thermodynamic temperature in the fixed point $(x, z)$ decrease.

- The maximum point of the stress $\sigma_{z z}$ gets large values when $\theta_{0}$ increases.

Figures 3 and 4 investigate how the conductive temperature $\phi$ and the in-plane displacement $u$ vary with all values of the two-temperature parameter. Note that the case of $a=0$ indicates the old situation (one-temperature 1TT) while the cases $a=0.2$ and $a=0.5$ indicate the two-temperature theory (2TT). Also, these figures show that:

- The two-temperature parameter has significant effect on all the field quantities.

- The waves reach the steady state depending on the value of the temperature discrepancy.

- It can be observed that $a>0$ corresponds a slower rate of decay for the temperature than the case when $a=0$.

- The behaviour of 2TT model may be differing than that of 1TT model near the boundary plane.

- The model of thermoelasticity with two temperatures predicts a finite speed of wave propagation that makes the generalized theory of thermoelasticity more agreeable with the physical properties of the material. 


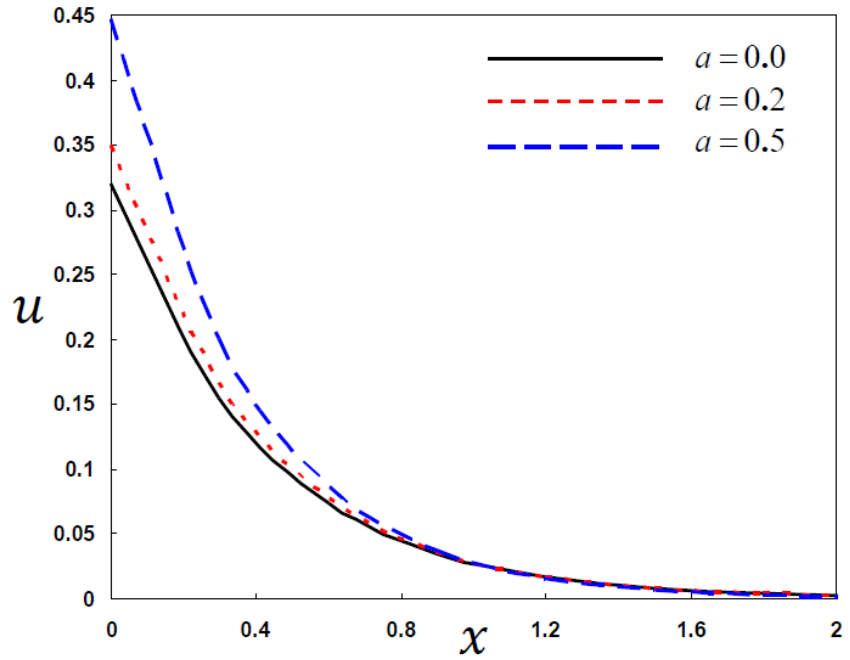

Figure 4. Variation of in-plane displacement $\mathbf{u}$ with distance $x$ for different values of the two-temperature parameter $a$.

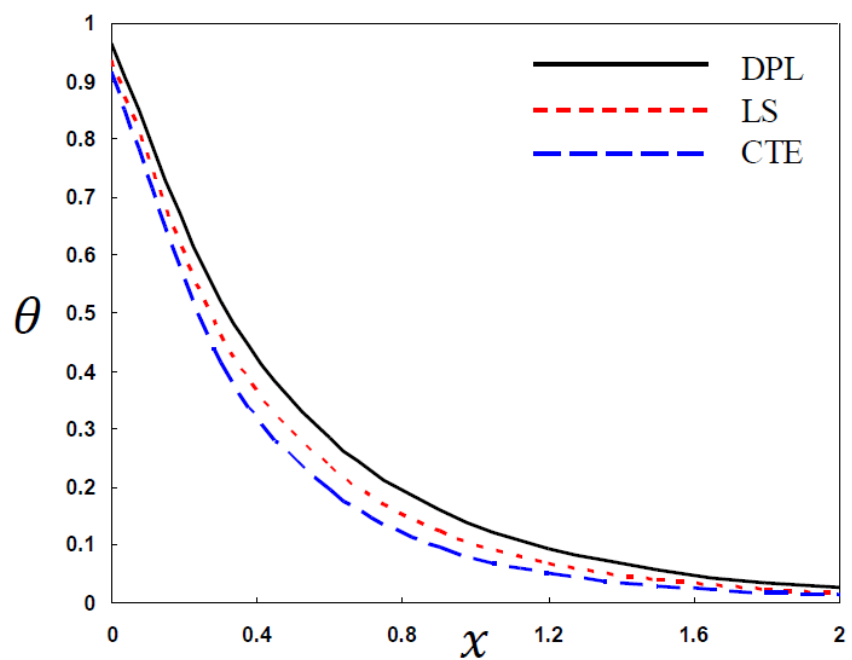

Figure 5. Variation of thermodynamic temperature $\theta$ with distance $x$ for different theories of thermoelasticity.

- The values of the conductive temperature decrease when the two-temperature parameter $a$ increases during all the running dimensionless time $t$.

- The maximum point of the displacement $u$ increases when $a$ increases.

Figures 5-7 plot comparison of the thermodynamic temperature $\theta$, the transverse displacement $w$, and the tangential shear stress $\sigma_{z x}$ against the direction $x$ for different values of PLs $\tau_{q}$ and $\tau_{\theta}$ at $z=1$ and different theories of thermoelasticity. The comparison of these figures shows the effect of PLs on the field variables. The field quantities including temperature, displacement, and stress components depend not only on space $x$ and time $t$, but also on the PLs $\tau_{\theta}$

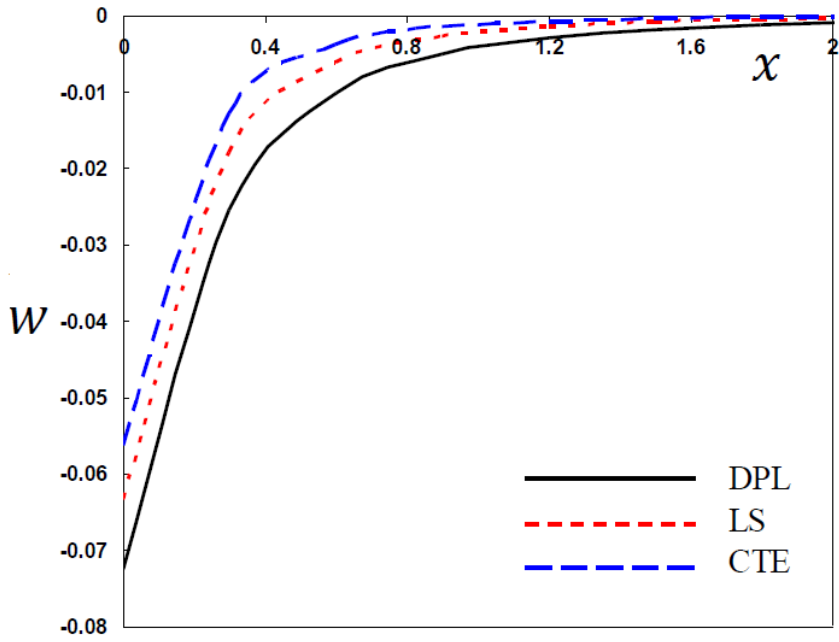

Figure 6. Variation of transverse displacement $w$ with distance $x$ for different theories of thermoelasticity.

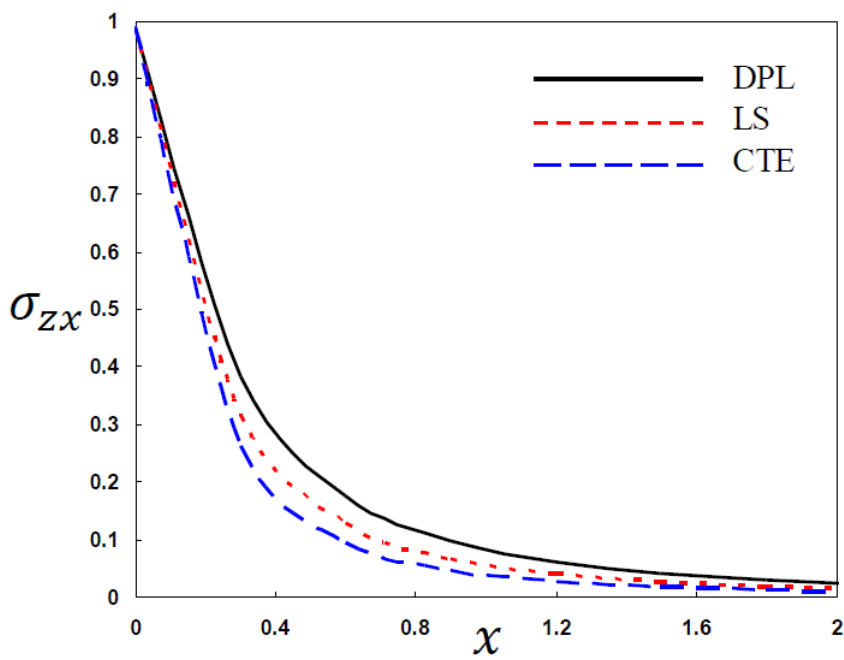

Figure 7. Variation of tangential shear stress $\sigma_{s z x}$ with distance $x$ for different theories of thermoelasticity.

and $\tau_{q}$. The computations were performed for one value of the dimensionless time, namely $t=0.15$ and various values of the parameters $\tau_{q}$ and $\tau_{\theta}$. These computations were carried out in the coupled theory (CTE) by setting $\left(\tau_{q}=\tau_{\theta}=0\right.$, $\delta=1)$, in Lord-Shulman (LS) theory by putting $\left(\tau_{\theta}=0\right.$, $\left.\tau_{q}=0.5, \delta=1\right)$ and in the generalized theory of thermoelasticity proposed by Tzou when $\tau_{q}=0.5 \geq \tau_{\theta}=0.2>0$. It is observed from these figures that:

- The values of the thermodynamic temperature $\theta$ and the stress component $\sigma_{z x}$ for CTE model are smaller compared to those for other theories whereas it is larger in the case of $w$.

- The distribution in LS theory is near to that in DPL theory and different to that in the CTE theory. 
- The values of $\tau_{q}$ and $\tau_{\theta}$ can judge whether the wavelike behaviour in the DPL heat conduction is dominant or not.

- The numerical results show that the PL parameters $\tau_{q}$ and $\tau_{\theta}$ may play a more important role in this task.

\section{Concluding remarks}

In this paper, an analytical solutions based on the normal mode analysis for the thermoelastic problem in solids have been developed and utilized. Analysis of in-plane displacement, tangential displacement, transverse normal stress, tangential shear stress, thermodynamic temperature, and conductive temperature due to mechanical load in a semi-infinite generalized thermoelastic medium is an interesting problem of mechanics. The generalized two-temperature theory of thermoelasticity in the context of dual phase lags (DPLs) model is used to solve this problem. The effect of the angle of inclination and phase-lags $\tau_{\theta}$ and $\tau_{q}$ as well as the two-temperature parameter on the field variables are investigated. The results concluded from the above analysis can be summarized as follows:

- The presence of phase-lags parameters plays a significant role in all the physical quantities.

- It is seen that the values of all the field variables are significantly dependent on the two-temperature parameter.

- According to the theory of thermoelasticity with two temperatures, we have to construct a new classification for materials according to their fractional parameter $a$ where this parameter becomes a new indicator of its ability to conduct heat under the effect of thermoelastic properties.

- It is also clear that the theories of coupled thermoelasticity and generalized thermoelasticity with one relaxation time can be obtained as limited cases.

- From our results, one can consider the theory of two-temperature generalized thermoelasticity as an improvement on studying elastic materials.

- The properties of a body depend largely on the direction of symmetry and the inclination of applied source.

- Significant difference in the values of the studied fields is noticed for different value of the angle of inclination.

Acknowledgements. This project was funded by the National Plan for Science, Technology and Innovation (MAARIFAH) King Abdulaziz City for Science and Technology - the Kingdom of Saudi Arabia - award number (12-NAN3083-03). The authors also, acknowledge with thanks Science and Technology Unit, King
Abdulaziz University for technical support.

Edited by: A. Barari

Reviewed by: three anonymous referees

\section{References}

Abouelregal, A. E. and Zenkour, A. M.: Generalized thermoelastic interactions due to an inclined load at a two-temperature halfspace, J. Theor. Appl. Mech., 54, 827-838, doi:10.15632/jtampl.54.3.827, 2016.

Abbas, I. A. and Zenkour, A. M.: Dual-phase-lag model on thermoelastic interactions in a semi-infinite medium subjected to a ramp-type heating, J. Comput. Theor. Nanosci., 11, 642-645, doi:10.1166/jctn.2014.3407, 2014a.

Abbas, I. A. and Zenkour, A. M.: Two-temperature generalized thermoelastic interaction in an infinite fiber-reinforced anisotropic plate containing a circular cavity with two relaxation times, J. Comput. Theor. Nanosci., 11, 1-7, doi:10.1166/jctn.2014.3309, 2014b.

Ailawalia, P. and Kumar, R.: Time harmonic inclined load in micropolar thermoelastic medium possessing cubic symmetry with one relaxation time, Tamk. J. Sci. Eng., 13, 117-126, 2010.

Allam, M. N., Elsibai, K. A., and Abouelregal, A. E.: Electromagneto-thermoelastic problem in a thick plate using Green and Naghdi theory, Int. J. Eng. Sci., 47, 680-690, doi:10.1016/j.ijengsci.2008.10.013, 2009.

Biot, M.: Thermoelasticity and irreversible thermodynamics, J. Appl. Phys., 27, 240-253, doi:10.1063/1.1722351, 1956.

Chandrasekharaiah, D. S.: Thermoelasticity with second sound, Appl. Mech. Rev., 39, 354-376, doi:10.1115/1.3143705, 1986.

Chen, P. J. and Gurtin, M.-E.: On a theory of heat conduction involving two temperatures, ZAMP Z. Angew Math. Phys., 19, 614-627, doi:10.1007/BF01594969, 1968.

Chen, P. J., Gurtin, M. E., and Willams, W. O.: On the thermodynamics of non-simple elastic material with two temperatures, ZAMP Z. Angew Math. Phys., 20, 107-112, doi:10.1007/BF01591120, 1969.

Cheng, J. C. and Zhang, S. Y.: Normal mode expansion method for laser generated ultrasonic lamb waves in orthotropic thin plates, Appl. Phys. B, 70, 57-63, doi:10.1007/s003400050008, 2000.

Green, A. E. and Lindsay, K. A.: Thermoelasticity, J. Elast., 2, 1-7, doi:10.1007/BF00045689, 1972.

Green, A. E. and Naghdi, P. M.: Thermoelasticity without energy dissipation, J. Elast., 31, 189-209, doi:10.1007/BF00044969, 1993.

Ignaczak, J. and Ostoja-Starzewski, M.: Thermoelasticity with Finite Wave Peeds, Oxford University Press, New York, p. 413, 2010.

Kumar, R. and Gupta, R. R.: Deformation due to inclined load in an orthotropic micropolar thermoelastic medium with two relaxation times, Appl. Math. Inform. Sci., 4, 413-428, 2010.

Kumar, R. and Rani, L.: Deformation due to inclined load in thermoelastic half-space with voids, Arch. Mech., 57, 7-24, 2005.

Lord, H. W. and Shulman, Y.: A generalized dynamical theory of thermoelasticity, J. Mech. Phys. Solid, 15, 299-309, doi:10.1016/0022-5096(67)90024-5, 1967.

Othman, M. I. A., Lotfy, Kh., and Farouk, R. M.: Effects of magnetic field and inclined load in micropolar thermoelastic medium 
possessing cubic symmetry under three theories, Int. J. Indust. Math., 1, 87-104, 2009.

Sharma, K.: Analysis of deformation due to inclined load in generalized thermodiffusive elastic medium, Int. J. Eng. Sci. Tech., 3, 117-129, 2011.

Tzou, D. Y.: A unified approach for heat conduction from macro-tomicro scales, J. Heat Transf., 117, 8-16, doi:10.1115/1.2822329, 1995a.

Tzou, D. Y.: Experimental support for the Lagging behavior in heat propagation, J Thermophys. Heat Transfer, 9, 686-693, doi: $10.2514 / 3.725,1995$ b.

Tzou, D. Y.: Macro-to Microscale Heat Transfer: the Lagging Behavior, 2nd Edition, Wiley, New York, p. 576, 2014.

Warren, W. E. and Chen, P. J.: Wave propagation in the two temperature theory of thermoelasticity, Acta. Mech., 16, 21-23, doi:10.1007/BF01177123, 1973.

Zenkour, A. M.: Three-dimensional thermal shock plate problem within the framework of different thermoelasticity theories, Compos. Struct., 132, 1029-1042, doi:10.1016/j.compstruct.2015.07.013, 2015.
Zenkour, A. M.: Two-dimensional coupled solution for thermoelastic beams via generalized dual-phase-lags model, Math. Model. Analys., 21, 319-335, doi:10.3846/13926292.2016.1157835, 2016.

Zenkour, A. M. and Abouelregal, A. E.: State-space approach for an infinite medium with a spherical cavity based upon twotemperature generalized thermoelasticity theory and fractional heat conduction, ZAMP Z. Angew Math. Phys., 65, 149-164, doi:10.1007/s00033-013-0313-5, 2014.

Zenkour, A. M. and Abouelregal, A. E.: Effects of phase-lags in a thermoviscoelastic orthotropic continuum with a cylindrical hole and variable thermal conductivity, Arch. Mech., 67, 457-475, 2015.

Zenkour, A. M., Mashat, D. S., and Abouelregal, A. E.: The effect of dual-phase-lag model on reflection of thermoelastic waves in a solid half space with variable material properties, Acta Mech. Solida Sinica, 26, 659-670, doi:10.1016/S0894-9166(14)600094, 2013. 